\title{
Lymphocele, CTCAE
}

National Cancer Institute

\section{Source}

National Cancer Institute. Lymphocele, CT CAE. NCI Thesaurus. Code C55705.

A disorder characterized by a cystic lesion containing lymph. 